\title{
Erosão dentária em paciente atleta: artigo de revisão
}

\author{
Dental erosion in an athlete: review article
}

\begin{abstract}
Bárbara Capitanio de Souza ${ }^{1}$
${ }^{1}$ Academia Brasileira de Odontologia do Esporte, Rio de Janeiro, RJ, Brasil

- A autora declara que não há conflito de interesse.
\end{abstract}

\section{Resumo}

Objetivo: discutir a erosão dentária, sua prevalência e fatores de risco, ressaltando a responsabilidade do cirurgião-dentista diante deste quadro patológico, principalmente frente ao atendimento de atletas. Material e Métodos: a pesquisa incluiu os artigos indexados na base de dados MEDLINE/PubMed. Foram considerados apenas os artigos publicados a partir do ano 2000. A pesquisa foi realizada utilizando as seguintes palavras e possíveis combinações: erosão dentária (dental erosion); erosão dentária, prevalência (dental erosion, prevalence); erosão dentária, atleta (dental erosion, athlete). Resultados: a erosão dentária é um processo químico multifatorial que resulta na perda de tecido dentário. A correta avaliação é fundamental para o diagnóstico da patologia e dos seus fatores causais. O paciente atleta está sujeito a um maior número de fatores de risco, indicando a necessidade de conceitos odontológicos preventivos adaptados ao campo da odontologia esportiva. Conclusão: é importante para o cirurgião-dentista reconhecer os sinais clínicos e os sintomas da erosão dentária, para restabelecer a saúde bucal do paciente atleta.

Palavras-chave: Erosão dentária; Saúde bucal; Fenômenos fisiológicos da nutrição esportiva.

\section{Abstract}

Objective: to discuss the prevalence and risk factors of dental erosion, highlighting the responsibility of a dental surgeon in the case of this pathological situation, particularly in front of the attendance of athletes. Material and Methods: the study included articles indexed in MEDLINE/PubMed database. Only articles published since 2000 were considered. The research was conducted using the following words and possible combinations: dental erosion; dental erosion, prevalence; dental erosion, and athlete. Results: dental erosion is a multifactorial chemical process that results in the loss of the dental tissue. The correct evaluation is fundamental for the diagnosis of the pathology and its causal factors. An athlete is prone to a greater number of risk factors, indicating the need for preventive dental measures adapted to the field of sports dentistry. Conclusion: it is important for a dental surgeon to recognize the clinical signs and symptoms of dental erosion for restoring the oral health of an athlete.

Keywords: Tooth erosion; Oral health; Sports nutritional physiological phenomena.

\section{Introdução}

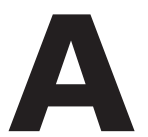
erosão dentária (ED) não teve maior destaque na literatura, nas últimas décadas, sendo pouco mencionada como um possível fator etiológico de perda de estrutura dentária. A abordagem deste tema teve maior destaque, nos últimos anos, devido ao aumento de sua frequência na população e à estreita relação que esta condição tem com o estilo e a qualidade de vida dos pacientes. ${ }^{1}$

A alteração é definida como perda progressiva e irreversível de estrutura dental provocada por processos químicos que não envolvam ação bacteriana. A ED tem sido associada a diversos fatores de risco como desordens alimentares, alterações sistêmicas e uso de medicações. ${ }^{2}$ Além disso, está ainda vinculada ao consumo excessivo de bebidas e alimentos com baixo $\mathrm{pH}$ e à atividade profissional em ambientes ácidos. ${ }^{3}$ A ocorrência de ED está aumentando e as lesões erosivas presentes progridem rapidamente, dando maior atenção à importância dos fatores desencadeantes do desgaste erosivo dente. ${ }^{4}$

Com as mudanças comportamentais e o estilo de vida, houve um aumento na prevalência do desgaste erosivo, especialmente entre os esportistas - profissionais, amadores ou mesmo as pessoas que aumentaram a prática de atividade física - o que coloca o tema em destaque na Odontologia clínica. Esta população está sujeita a um maior número de fatores de risco combinados, como hábitos alimentares e comportamentais. ${ }^{5}$ Adicionalmente, o fluxo salivar pode assumir um papel igualmente importante, pois quando o seu valor esta reduzido, o que acontece muito durante a atividade esportiva, a lubrificação e o efeito tampão na cavidade oral poderão estar alterados, facilitando a ocorrência de desmineralização por erosão. ${ }^{6}$

É importante para o cirurgião-dentista reconhecer os sinais clínicos e os sintomas da ED, objetivando restabelecer a saúde bucal ao paciente. Para isto, a informação a respeito do assunto é imprescindível, capacitando o profissional. A proposta deste artigo é discutir a erosão dentária, sua prevalência e fatores de risco, ressaltando a responsabilidade do cirurgião-dentista diante deste quadro patológico, principalmente frente ao atendimento de atletas.

\section{Material e Métodos}

Trata-se de um estudo descritivo analítico, que foi realizado através de revisão de literatura. A pesquisa foi desenvolvida em fevereiro de 2017 e incluiu os artigos indexados na base de dados MEDLINE/PubMed. Para uma leitura mais atual, foram considerados apenas os artigos publicados a partir do ano 2000. A busca de artigos foi realizada utilizando as seguintes palavras e possíveis combinações: erosão dentária (dental erosion); erosão dentária, prevalência (dental erosion, prevalence); erosão dentária, atleta (dental erosion, athlete); Foram excluídos os artigos que não apresentavam resumo disponível ou não tratavam do tema proposto. 


\section{Revisão de Literatura}

A ED inicia com a uma desmineralização das camadas mais superficiais do esmalte, evoluindo para uma maior perda da estrutura dental. Qualquer substância ácida com $\mathrm{pH}$ inferior ao crítico para o esmalte $(5,5)$ e a dentina $(6,5)$ pode resultar em perda de estrutura dentária. O desenvolvimento do processo erosivo começa com um amolecimento da superfície do dente e posterior dissolução dos seus elementos progressivamente. ${ }^{7}$ Este quadro pode ter uma maior ou menor intensidade, dependendo da capacidade de tamponamento da saliva e da disponibilidade de flúor. Tratase de um processo químico que não envolve a influência de bactérias. $^{8}$

O diagnóstico de formas precoces de erosão é difícil, pois é acompanhado por poucos sinais e sintomas. A erosão inicial do esmalte não provoca alteração da cor ou suavização da superfície do dente, sendo difícil um diagnóstico por exame clínico. Os sintomas no doente, nestes estádios iniciais, são frequentemente ausentes ou muito limitados. A progressão posterior da erosão oclusal leva a um arredondamento das cúspides e as restaurações ficam acima do nível das superfícies dentárias adjacentes. Em casos graves, a morfologia oclusal pode desaparecer (Figura 1). ${ }^{2}$
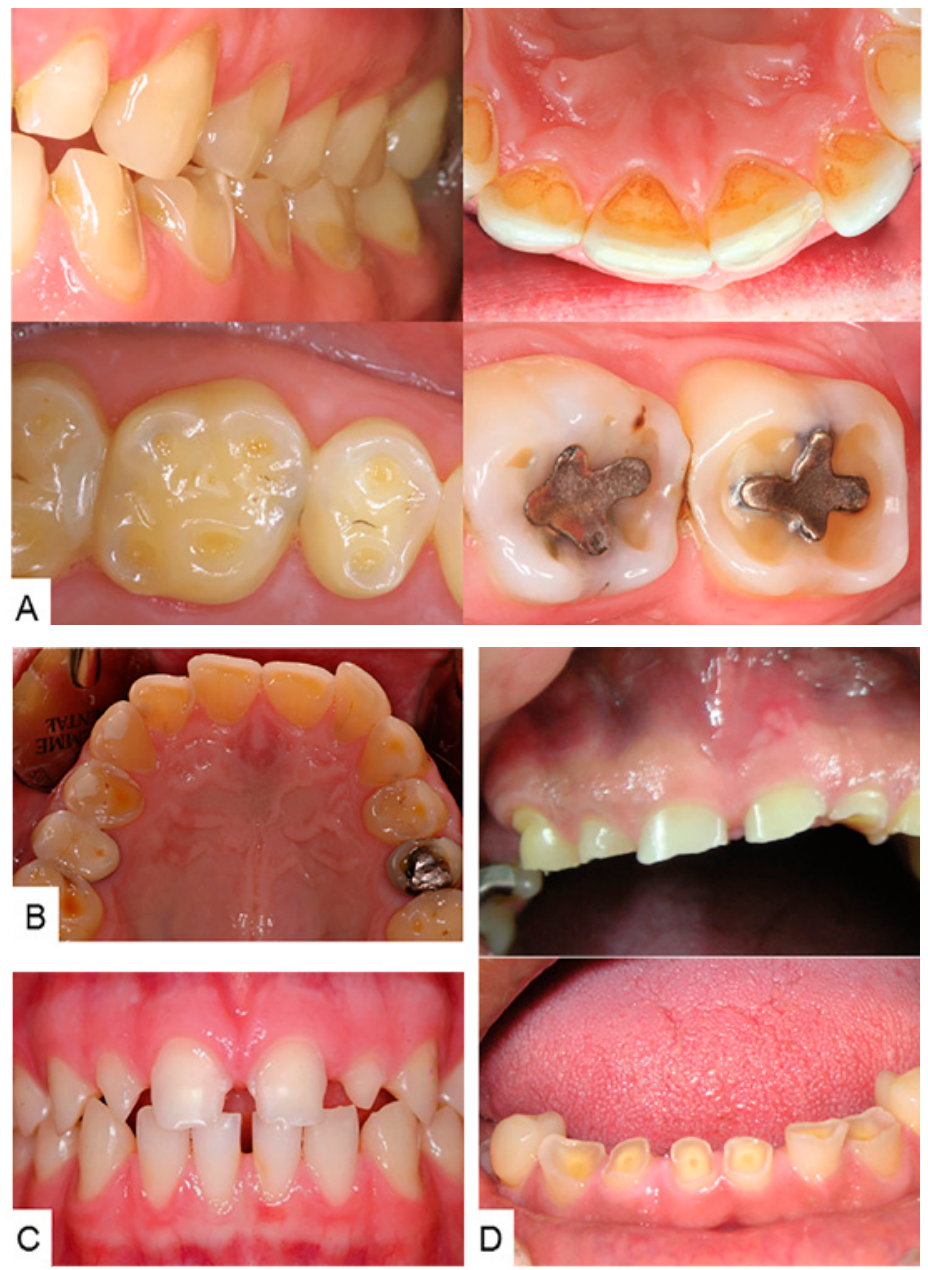

Figura 1. Aspecto clínico de lesões erosivas. (A) Perda do esmalte nas faces vestibulares e palatinas com início de exposição dentinária. Progressão da erosão oclusal, apresentando um arredondamento das cúspides dos dentes molares e evidenciando as restaurações que estão acima do nível das superfícies dentárias adjacentes; (B) perda de tecido dentário vista até a margem gengival, com exposição dentinária, decorrente de transtorno alimentar relacionado à bulimia; (C) perda do esmalte dentário devido ao consumo excessivo de bebidas ácidas; (D) dentes apresentando alteração da macromorfologia ocorrida pelo processo de erosão química devido a distúrbios gástricos

Fonte: Adaptado de: (A) Lussi A, Schaffner M, Jaeggi T. Diagnosis of dental erosions. Swiss Dent J. 2016;126(5):466-7. (B) Bruno V, Amato M, Catapano S, lovino P. Dental erosion in patients seeking treatment for gastrointestinal complaints: a case series. J Med Case Rep. 2015;9:250. (C) Al-Salehi SK. Restorative management of intrinsic and extrinsic dental erosion. j indian prosthodont Soc. 2014;14(Suppl 1):215-21. (D) Paryag A, Rafeek R. Dental erosion and medical conditions: na overview of aetiology, diagnosis and management. West Indian Med J. 2014;63(5):499-502.

A aparência clínica é a característica mais importante para profissionais de Odontologia diagnosticar a ED, o que é de particular importância para as ações de prevenção e tratamento da condição. ${ }^{13}$ Algumas alterações são identificadas como uma concavidade no esmalte, cuja largura tem maior dimensão do que a profundidade. A perda da estrutura dentária na face vestibular deve ser distinguida das lesões em forma de cunha, localizadas próximas à junção esmalte-cemento, causadas por outros processos (Tabela 1). ${ }^{14}$ Diferentes escalas têm sido utilizadas em diferentes sistemas para o exame clínico e o diagnóstico do processo erosivo (Tabelas 2 e 3). ${ }^{15,16}$

Tabela 1. Critérios de classificação do desgaste erosivo

\begin{tabular}{c|c}
\hline Grau & Descrição \\
\hline 0 & Nenhum desgaste erosivo do dente. \\
\hline 1 & Perda inicial da textura da superfície. \\
\hline 2 & $\begin{array}{c}\text { Distinto defeito; Perda de tecido duro envolvendo } \\
<50 \% \text { da área da superfície dentária. }\end{array}$ \\
\hline 3 & $\begin{array}{c}\text { Perda de tecido duro envolvendo } \geq 50 \% \text { da } \\
\text { área da superfície dentária. }\end{array}$ \\
\hline
\end{tabular}

Nas pontuações 2 e 3 a dentina pode estar envolvida. Adaptado de: Carvalho TS, Colon P, Ganss C, Huysmans MC, Lussi A, Schlueter N, et al. Consensus Report of the European Federation of Conservative Dentistry: erosive tooth wear - diagnosisand management. Swiss Dent J. 2016;126(4):342-6

Tabela 2. Escala ordinal utilizada para a classificação de coberturas nas superfícies oclusais dos primeiros molares decíduos e permanentes

\begin{tabular}{c|c}
\hline Grau & Descrição \\
\hline 0 & Sem ponta de cúspide intacta \\
\hline 1 & Ponta arredondada da cúspide \\
\hline 2 & Cupping $\leq 1 \mathrm{~mm}$ \\
\hline 3 & Cupping $>1 \mathrm{~mm}$ \\
\hline 4 & $\begin{array}{c}\text { Cofragens fundidas: pelo menos duas cupping são } \\
\text { fundidas junto no mesmo dente }\end{array}$ \\
\hline
\end{tabular}

Nas superfícies oclusais, as lesões iniciais podem se apresentar como pequenos pontos socavados, mais comumente nas pontas de cúspides. Com a progressão da lesão, esta assume uma forma que se assemelha a uma xícara, conhecida como cupping. Adaptado de: Johansson AK, Lingström P, Imfeld T, Birkhed D. Influence of drinking method on toothsurface $\mathrm{pH}$ in relation to dental erosion. Eur J Oral Sci. 2004;112(6):484-9 
Tabela 3. Escala ordinal utilizada para avaliar a gravidade da erosão dentária nas superfícies vestibular e lingual dos dentes anteriores superiores

\begin{tabular}{|c|c|}
\hline Grau & Descrição \\
\hline 0 & Sem mudanças visíveis, estruturas de desenvolvimento permanecem, macromorfologia intacta. \\
\hline 1 & $\begin{array}{c}\text { Esmalte suavizado, as estruturas de desenvolvimento estão total ou parcialmente desaparecidas. } \\
\text { A superfície do esmalte é brilhante, mate, irregular, "derretida", arredondada ou plana, e macromorfologia geralmen- } \\
\text { te intacta. }\end{array}$ \\
\hline 2 & $\begin{array}{l}\text { Superfície de esmalte como descrito no grau } 1 . \\
\text { Macromorfologia com formação de concavidade dentro do esmalte, sem exposição dentinária. }\end{array}$ \\
\hline 3 & $\begin{array}{l}\text { Superfície do esmalte descrita nos graus } 1 \text { e } 2 \text {. } \\
\text { A macromorfologia mudou muito (perto da exposição dentinária de grandes superfícies) ou a superfície da dentina } \\
\text { foi exposta } \leq 1 / 3 .\end{array}$ \\
\hline 4 & 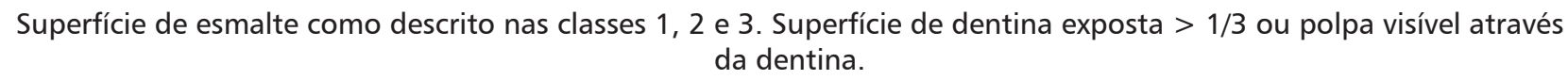 \\
\hline
\end{tabular}

Adaptado de: Hasselkvist A, Johansson A, Johansson AK. A 4 year prospective longitudinal study of progression of dental erosion associated to life style in 13-14year-old Swedish adolescents. J Dent. 2016;47:55-62

Além da ED, existem outras condições que também envolvem lesões dentárias não cariosas e caracterizam-se por ocasionar desgaste na estrutura dentária na ausência de micro-organismos (Figura 2). A atrição pode ser definida como a ação de desgaste físico dente contra dente, que pode ocorrer durante a mastigação, a deglutição e a fala ou, ainda, pode ser observada em associação com outras condições, como desordens temporomandibulares. ${ }^{17} \mathrm{~A}$ abfração é uma perda patológica de tecido dental ocasionada por forças traumáticas induzidas por pressão excessiva sobre os dentes. Ocorre normalmente na região cervical dos elementos dentários e não está associada às doenças periodontais. As lesões são mais profundas e podem se estender para a margem subgengival. ${ }^{18} \mathrm{~A}$ abrasão é caracterizada como um desgaste patológico ocasionado pela ação de processos mecânicos que envolvem substâncias ou objetos. As lesões por abrasão apresentam superfície dura e polida, podendo ser observadas ranhuras na estrutura dentária. As lesões são mais frequentes nas regiões cervicais e vestibulares dos dentes e costumam ser mais superficiais. ${ }^{19}$
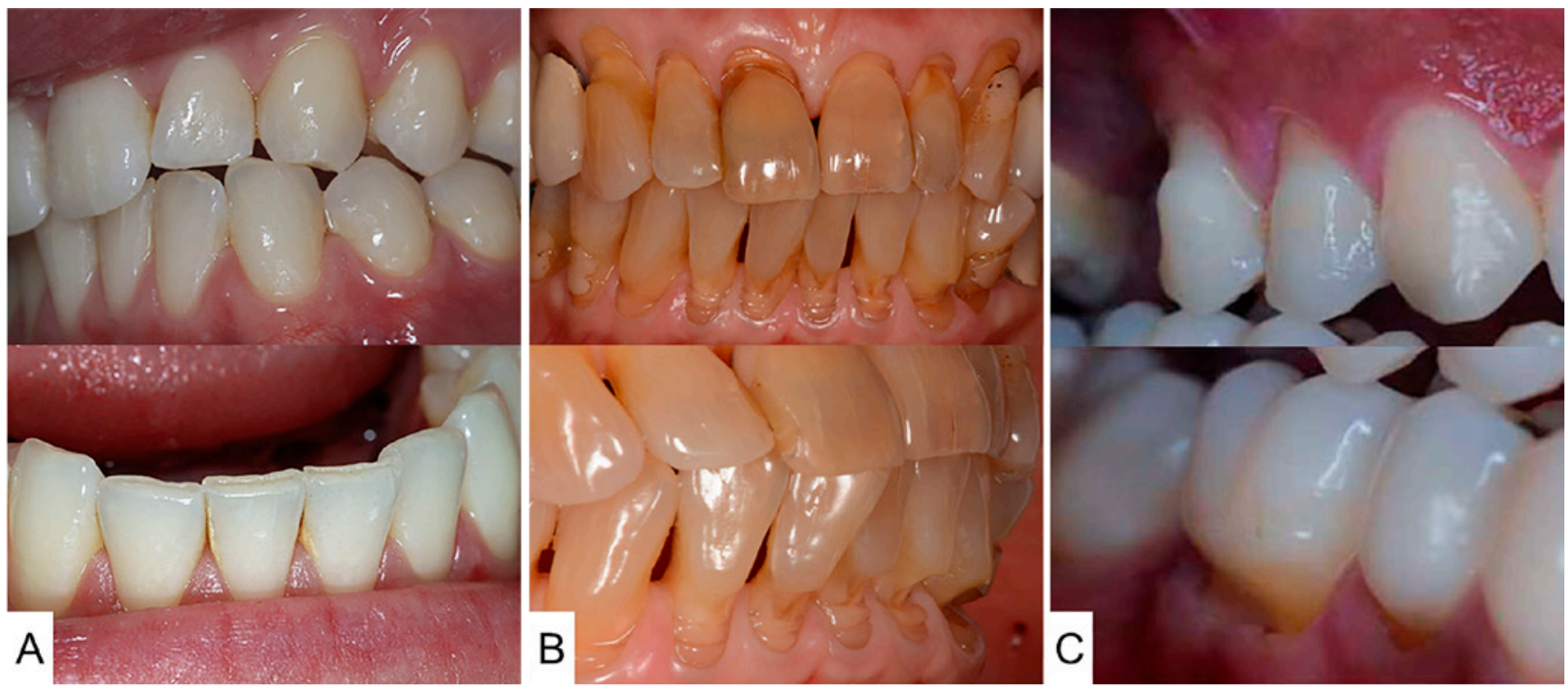

Figura 2: Aspecto clínico de lesões não cariosas. (A) Atrição: presença de facetas de desgaste por atrito entre os dentes opostos. Observam-se desgaste da cúspide do canino superior e exposição da dentina na região incisal dos dentes incisivos inferiores. (B) Abfração: lesões em forma de cunha na região cervical dos dentes, com perda significativa de tecido dentário. (C) Abrasão: desgaste dentário cervical e recessão gengival causada por escovação traumática

Fonte: Adaptado de: (A) Jonsgar C, Hordvik PA, Berge ME, Johansson AK, Svensson P, Johansson A. Sleep bruxism in individuals with and without attrition-type tooth wear: Na exploratory matched case-control electromyographic study. J Dent. 2015;43(12):1504-10. (B) Nascimento MM, Dilbone DA, Pereira PN, Duarte WR, Geraldeli S, Delgado AJ. Abfraction lesions: etiology, diagnosis, and treatment options. 
A prevalência da ED vem aumentando nos últimos anos e apresenta-se de forma variável entre as idades, sendo mais significativa em jovens e adultos. ${ }^{8}$ Ocorre com o aumento da idade dos pacientes, uma vez que a evolução do processo degenerativo da ED se dá sobre a influência do tempo em que os fatores atuam sobre os dentes. Entretanto, estudos longitudinais indicam um aumento da erosão do tecido dentário, cada vez mais significativo, em populações mais jovens; além disso, a extensão e gravidade das lesões também apresentam maior evolução ao longo dos anos. ${ }^{20,21} \mathrm{~A}$ erosão no tecido dentinário também foi mais evidente nos estudos recentes. ${ }^{22}$

Os estudos epidemiológicos, em sua maioria, são recentes e de caráter transversal, sendo realizados principalmente a partir da década de $90 .{ }^{23,24}$ Os estudos de prevalência indicam a proporção de indivíduos com erosão em um determinado período. Para melhor conhecer a progressão da ED e desenvolver planos preventivos e medidas de tratamento são necessários estudos sobre a avaliação da incidência do processo erosivo. Além disso, em estudos epidemiológicos, muitas vezes não é possível diferenciar os tipos de desgastes dentários. $^{8}$

\section{Discussão}

A erosão dentária ocupacional é compreendida como o efeito destrutivo ou de perda da estrutura dentária decorrente da ação direta ou indireta da atividade laboral do indivíduo. Trabalhadores de laboratórios e certas indústrias como as de minerais e químicas são mais expostos a substâncias ácidas e costumam apresentar lesões decorrentes da ED. Profissionais que atuam como degustadores de alimentos e bebidas também podem apresentar ED devido à frequência de exposição aos alimentos ácidos, como o que ocorre com enólogos. ${ }^{25} \mathrm{Da}$ mesma forma, o profissional atleta também está sujeito aos riscos da ED, devido sua exposição a diferentes fatores relacionados com o processo de erosão.

Com a evolução da ED, podem ser observadas alterações mais pronunciadas na macromorfologia dentária. A condição será então mais fácil de ser reconhecida e o paciente apresentará mais sintomas e queixas, assim como afetar a qualidade de vida relacionada à saúde bucal. ${ }^{26}$ Neste sentido, para o paciente atleta, o reconhecimento precoce da ED e de seus fatores de risco são importantes para o desenvolvimento de ações que objetivam evitar, também, qualquer possível intercorrência no desempenho de sua prática esportiva.

Em relação a estes pacientes, de maneira geral, observase uma maior prevalência de doenças orais. ${ }^{27} \mathrm{~A}$ associação entre a atividade esportiva e o desgaste erosivo foi bastante destacada. ${ }^{28} \mathrm{~A}$ atividade erosiva sobre a superfície dentária apresenta prevalência alta e variável (em média de 36\% a $85 \%$ ), possivelmente associada ao comportamento alimentar do atleta e às modalidades de esporte praticado. ${ }^{29}$ Um estudo com atletas olímpicos, encontrou uma forte prevalência de ED $(44,6 \%)$ nos esportistas examinados. As regiões mais afetadas, com uma classificação de moderada a severa, foram as dos dentes anteriores, com $37,6 \%$ dos casos, e as dos dentes posteriores, com $48 \% .^{30}$

O processo que envolve a perda da estrutura dentária pela erosão possui característica multifatorial, podendo ocorrer a ação simultânea de diferentes fatores. A etiologia complexa da ED justifica a variação na ocorrência, distribuição e gravidade das lesões, sendo que os fatores etiológicos envolvidos podem ter origem intrínseca ou extrínseca. ${ }^{1}$ Enquanto que os fatores intrínsecos estão relacionados com as alterações biológicas individuais, os fatores extrínsecos envolvem, de modo geral, componentes relacionados ao comportamento e estilo de vida (Tabela 4$)^{2}{ }^{2}$

Tabela 4. Principais fatores de risco para a erosão dentária no paciente atleta

\begin{tabular}{|c|c|c|}
\hline & Fatores de Risco & Considerações \\
\hline \multirow{3}{*}{ Intrínsecos } & Saliva & $\begin{array}{l}\text { A saliva protege o esmalte da ED através de vários mecanismos. A capacidade tampão da saliva pode ser modifi- } \\
\text { cada pela alteração da composição e fluxo salivar. A saliva também atua como uma barreira de difusão ou película } \\
\text { aderida, prevenindo o contato direto entre os ácidos e a superfície dos dentes. }\end{array}$ \\
\hline & Fatores biológicos & $\begin{array}{l}\text { Além da saliva e da película aderida, a posição relativamente aos tecidos moles e à língua desempenham um papel } \\
\text { no desenvolvimento da ED, pois podem influenciar o padrão de retenção/depuração de agentes erosivos. }\end{array}$ \\
\hline & Doenças & $\begin{array}{l}\text { Transtornos alimentares de ordem comportamental, como a anorexia e a bulimia, têm efeitos sobre a saúde oral. } \\
\text { Atletas com estes distúrbios expõe a estrutura dentária ao contato repetido com fluido ácido estomacal. Este com- } \\
\text { portamento pode estar relacionado com a necessidade de manutenção do peso dentro da modalidade esportiva. }\end{array}$ \\
\hline \multirow{4}{*}{ Extrínsecos } & $\begin{array}{l}\text { Hábitos } \\
\text { alimentares }\end{array}$ & $\begin{array}{l}\text { A quantidade, a frequência, a textura e a forma como os alimentos e bebidas com maior acidez são introduzidos } \\
\text { na cavidade oral vão determinar o desafio erosivo e o padrão do desgaste dentário. Esse fator está relacionado } \\
\text { com as exigências nutricionais e calóricas da preparação física e da modalidade do esporte praticada. }\end{array}$ \\
\hline & $\begin{array}{l}\text { Hábitos } \\
\text { comportamentais }\end{array}$ & $\begin{array}{l}\text { Envolvem uma sobreposição dos efeitos relacionados com a alimentação e a suplementação nutricional, a rotina } \\
\text { de treinamento e a prática da modalidade esportiva. O estilo de vida saudável do paciente atleta, que envolve uma } \\
\text { rotina de treinos e uma alimentação diferenciada, pode ser paradoxal, quando consideramos a ED. }\end{array}$ \\
\hline & $\begin{array}{l}\text { Intensidade } \\
\text { dos treinos e } \\
\text { modalidade } \\
\text { esportiva }\end{array}$ & $\begin{array}{l}\text { Pode ocorrer uma diminuição do fluxo salivar relacionada com um aumento de atividade simpática, durante o } \\
\text { exercício intenso. Ainda, a perda de líquidos durante a prática esportiva envolve uma redução de eletrólitos e dimi- } \\
\text { nuição da função de tamponamento dos íons cálcio e fluoreto contidos na saliva, contribuindo para a progressão } \\
\text { da ED. Dentro da modalidade esportiva, os nadadores pertencem a um grupo de risco. As técnicas de desinfecção, } \\
\text { como a cloração a gás e hipoclorito de sódio, modificam o pH da água. }\end{array}$ \\
\hline & Medicações & $\begin{array}{l}\text { A qualidade e a quantidade do luxo salivar podem sofre alterações decorrentes do uso de alguns medicamentos. } \\
\text { Muitos atletas possuem autorização para o uso de medicações restritas, sendo importante o conhecimento sobre } \\
\text { seus efeitos. }\end{array}$ \\
\hline
\end{tabular}


A saliva possui características e funções importantes para o controle da ED. Ela possui ação física de lavagem do meio bucal, auxiliando na redução de retenção de produtos ácidos, e age como uma película de proteção da superfície dentária, reduzindo o contato de agentes erosivos com o dente. Também, possui ação química referente à sua capacidade tampão, mantendo o seu $\mathrm{pH}$ constante em meio ácido. ${ }^{31}$ Portanto, é fundamental a manutenção da qualidade e quantidade do fluxo salivar, para que essas funções estejam atuando plenamente. Os fatores externos como hábitos alimentares e comportamentais, intensidade e frequência de treinos, modalidade do esporte desenvolvida e o uso de medicações podem agir de forma direta ou indireta sobre o fator salivar e o desafio erosivo sofrido pelas estruturas dentárias. ${ }^{32}$

Dentre uma grande variedade de alimentos, as bebidas esportivas aparecem como agentes potencializadores da ED. São artigos utilizados para reposição de água, eletrólitos e níveis energéticos, de baixo $\mathrm{pH}$, contendo frequentemente ácido cítrico em sua composição, o qual é altamente erosivo, prolongando o efeito de desmineralização. ${ }^{5} \mathrm{~A}$ frequência, a quantidade e a forma de ingestão de produtos erosivos influenciam na intensidade e gravidade das lesões. ${ }^{8}$ Líquidos ácidos, por exemplo, possuem maior potencial de velocidade e cobertura de contato da superfície dentária, o que aumenta ainda mais o risco de ED para o paciente atleta.

Adicionalmente, algumas características individuais, envolvendo o volume e posicionamento dos tecidos orais, podem influenciar na retenção e depuração de agentes agressivos aos tecidos dentários. ${ }^{2} \mathrm{O}$ paciente atleta pode apresentar uma maior hipertonicidade e hipertrofia da musculatura da face, especialmente do músculo masseter, decorrente das forças de apertamento dentário realizado durante o esforço físico. ${ }^{33,34}$ Devemos também considerar, o possível uso de moduladores de crescimento, que podem alterar o desenvolvimento musculoesquelético da face. Fatores como estresse excessivo e prolongado, síndrome de Sjögren, diabetes e doenças das glândulas salivares, também podem ocasionar modificações do perfil salivar. Distúrbios alimentares, hiperacidez estomacal e doença do refluxo gastresofágico irão atuar mais especificamente sobre a estrutura dentária, no processo de desmineralização. ${ }^{2,8}$

Além disso, pacientes atletas com necessidades especiais podem apresentar algumas doenças ou síndromes que manifestam, dentro de suas características clínicas, alterações no fluxo salivar, hipoplasia dos tecidos dentários e macroglossia. Todos estes fatores aumentam o risco de ED devem ser avaliados. Muitos atletas possuem autorização para o uso de medicações restritas, sendo importante o conhecimento sobre seus efeitos. As principais medicações que podem influenciar no desafio erosivo, pois podem modificar a produção salivar, pertencem aos grupos dos antiespasmódicos, anticolinérgicos, anti-histamínicos, antieméticos, antineoplásicos, ansiolíticos, broncodilatadores, anti-hipertensores, diuréticos e antiarrítmicos. ${ }^{35,36}$

Outros importantes fatores intrínsecos, apontados na literatura e relacionados com o processo de ED, são os distúrbios gástricos, como o refluxo gastroesofágico, e os transtornos alimentares. As consequências destas condições mostram-se bastante nocivas para os tecidos dentários. A exposição crônica ao ácido gástrico, ocasionada pelas patologias, pode causar lesões em tecidos duros e moles da cavidade oral. ${ }^{37} \mathrm{O}$ diagnóstico da causa da ED pode ser complexo, nestes casos. A presença de erosão na face lingual e na incisal dos dentes anteriores inferiores permite sugerir a indicação destas alterações - distúrbios gástricos e transtornos alimentares -, como possíveis causas. Assim, é fundamental que, previamente ao exame clínico, seja realizada uma anamnese detalhada, para identificar a principal causa das lesões e direcionar adequadamente táticas preventivas e abordagens terapêuticas corretas. ${ }^{38}$

Considerando, também, a intensidade e frequência de treinos e a modalidade do esporte desenvolvida, podemos pontuar outras situações que envolvem maior risco para a ED. Dependendo do modelo de estresse a que o corpo é submetido, podem ocorrer situações fisiológicas diversas. Em caso de treinamento intenso, é observado um aumento na atividade do sistema nervoso simpático, promovendo vasoconstrição de vasos sanguíneos e estruturas de glândulas salivares, reduzindo o fornecimento de água para a produção de saliva. A liberação de noraepinefrina, nestas condições, também está associada à baixa de fluxo salivar. ${ }^{39-41} \mathrm{O}$ ambiente de treinamento também tem ação sobre a ED. A prática da natação em piscinas é considerada como um fator de risco para a desmineralização dentária. ${ }^{42}$ Isso ocorre devido a um desequilíbrio do $\mathrm{pH}$ da água, pelo uso de agentes clorados, que são utilizados para a realização do processo de sanitização deste meio. ${ }^{43}$

\section{Orientações para a Prática Clínica}

A ED é um processo que ocorre naturalmente na cavidade bucal, ao longo da vida. Contudo, para um adequado controle da condição, é necessária a distinção entre os processos patológicos e fisiológicos que envolvem a perda de tecido dentário. Uma correta identificação da progressão da ED é fundamental, pois determinará as medidas preventivas e intervencionistas necessárias para cada caso; entretanto, uma das dificuldades na avaliação clínica é a sobreposição fatores causais, principalmente quando o processo de erosão está em um estágio mais avançado. ${ }^{2}$

O diagnóstico e monitorização do processo de ED devem ser realizados cuidadosamente, utilizando-se do maior número de ferramentas disponíveis, para a coleta de informações, especialmente em caso de paciente atleta, uma vez 
que estes estão sujeitos a um maior número de fatores de risco. A avaliação clínica para diagnóstico e controle das lesões pode ser realizada com o auxílio de modelos de estudo e fotografias. ${ }^{14}$ Também é recomendada a realização de um odontograma com a indicação de um índice clínico de ED, que viabilize a comparação futura do exame. Além disso, para que todos os possíveis fatores de risco sejam avaliados, é fundamental o conhecimento da rotina do paciente atleta. Sendo assim, pode ser realizado um diário contemplando os fatores etiológicos intrínsecos e extrínsecos, já abordados. $\mathrm{O}$ prontuário de avaliação do processo de ED deve ser revisado e atualizado como rotina, já que este processo é dinâmico. Quando aplicável, também podem ser realizados exames para avaliação de qualidade e fluxo salivar.

Com base nessas análises, pode ser realizado um programa específico para a ED, visando a prevenção ou o tratamento de lesões já estabelecidas. Em relação aos hábitos alimentares e à suplementação nutricional, é importante destacar a comunicação com a equipe responsável pelo treinamento do atleta e seu nutricionista. Não cabe ao cirurgião-dentista a intervenção direta sobre a dieta do atleta, pois este está seguindo uma prescrição profissional. Neste caso, o cirurgião-dentista deve esclarecer o profissional responsável pela dieta do esportista e buscar alternativas para a prevenção e a redução dos danos do processo de erosão.

A evidência sobre a orientação de evitar a escovação dentária imediatamente após a ingestão de alimentos ácidos é limitada; portanto, devem ser mantidas as orientações para a prevenção da cárie. Além disso, a escovação é um veículo para o fornecimento de flúor, o qual tem ação na redução da solubilidade dos tecidos dentários. O uso de escova de dente macia e creme dental de baixa abrasividade são orientações importantes adicionais, para reduzir o desgaste dos dentes. ${ }^{44}$
Compostos fluoretados e produtos com propriedades remineralizantes podem favorecer a recuperação mineral das superfícies dentais desmineralizadas pela ED. O flúor é o principal agente remineralizante indicado para a prevenção e o controle do processo de erosão, aumentando a resistência superficial a partir da recuperação mineral. O uso de dentifrícios e soluções para bochechos podem ser uma fonte de exposição aos fluoretos e se mostram efetivos no controle da ED. ${ }^{45} \mathrm{~A}$ utilização de vernizes fluoretados também resulta em aumento da resistência da superfície dental exposta a agentes erosivos e pode ser uma alternativa efetiva e conservadora para o tratamento e controle da ED, reduzindo a perda da estrutura dentária e a sensibilidade. ${ }^{46}$

O tratamento restaurador, para os casos de ED, também tem como objetivo reduzir a dor e o desconforto causado pela hipersensibilidade dentinária, além de interromper a progressão das lesões e reestabelecer a estética e a função. $O$ tratamento restaurador não pode ser utilizado isoladamente, devendo ser precedido ou usado em conjunto com estratégias preventivas, para melhor efetividade do tratamento. A escolha dos materiais restauradores assim como as técnicas para reabilitação devem considerar as particularidades do paciente atleta e da modalidade de esporte praticada. ${ }^{14}$

\section{Conclusão}

A ED é um processo químico multifatorial que resulta na perda de tecido dentário. A correta avaliação de cada caso é fundamental para o diagnóstico da patologia e dos seus fatores causais, permitindo o tratamento adequado. $\mathrm{O}$ paciente atleta está sujeito a um maior número de fatores de risco, indicando a necessidade de conceitos odontológicos preventivos adaptados ao campo da odontologia esportiva.

\section{Referências}

1. Hamasha AAH, Zawaideh FI, Al-Hadithy RT. Risk Indicators Associated With Dental Erosion Among Jordanian School Aged 12-14 Years of Age. Int J Paediatr Dent. 2014;24(1):56-68.

2. Lussi A, Jaeggi T. Erosion-diagnosis and risk factors. Clin Oral Investig. 2008;12(Suppl 1):5-13.

3. Grippo JO, Simring M, Schreiner S. Attrition, abrasion, corrosion and abfraction revisited: a new perspec $\neg$ tive on tooth surface lesions. J Am Dent Assoc. 2004;135:1109-18.

4. Little JW. Eating disorders: dental implications. Oral Surg Oral Med Oral Pathol Oral Radiol Endod. 2002;93:138-43.

5. Harpenau LA, Noble WH, Kao RT. Diagnosis and Management of Dental Wear. J Calif Dent Assoc. 2011;39(4):225-31.

6. Barron RP, Carmichael RP, Marcon MA, Sandor GK. Dental erosion in gastroesophageal reflux disease. J Can Dent Assoc. 2003;69:84-9.

7. Chikte UM, Naidoo S, Kolze TJ, Grobler SR. Patterns of tooth surface loss among winemakers. SADJ. 2005;60:370-4.

8. Johansson AK, Omar R, Carlsson GE, Johansson A. Dental erosion and its growing importance in clinical practice: from past to present. Int $\mathrm{J}$ Dent. 2012:632907.

9. Lussi A, Schaffner M, Jaeggi T. Diagnosis of dental erosions. Swiss Dent J. 2016;126(5):466-7.

10. Bruno V, Amato M, Catapano S, Iovino P. Dental erosion in patients se- eking treatment for gastrointestinal complaints: a case series. J Med Case Rep. 2015;9:250.

11. Al-Salehi SK.

12. Restorative management of intrinsic and extrinsic dental erosion. J Indian Prosthodont Soc. 2014;14(Suppl 1):215-21.

13. Paryag A, Rafeek R. Dental erosion and medical conditions: na overview of aetiology, diagnosis and management. West Indian Med J. 2014;63(5):499-502.

14. Lussi A. Erosive tooth wear - a multifactorial condition of growing concern and increasing knowledge. Monogr Oral Sci. 2006;20:1-8.

15. Carvalho TS, Colon P, Ganss C, Huysmans MC, Lussi A, Schlueter N, et al. Consensus Report of the European Federation of Conservative Dentistry: Erosive tooth wear - diagnosisand management. Swiss Dent J. 2016;126(4):342-6.

16. Johansson AK, Lingström P, Imfeld T, Birkhed D. Influence of drinking method on tooth-surface $\mathrm{pH}$ in relation to dental erosion. Eur J Oral Sci. 2004;112(6):484-9.

17. Hasselkvist A, Johansson A, Johansson AK. A 4 year prospective longitudinal study of progression of dental erosion associated to lifestyle in 13-14year-old Swedish adolescents. J Dent. 2016;47:55-62.

18. Jonsgar C, Hordvik PA, Berge ME, Johansson AK, Svensson P, Johansson A. Sleep bruxism in individuals with and without attrition-type tooth wear: Na exploratory matched case-control electromyographic study. J Dent. 2015;43(12):1504-10. 
19. Nascimento MM, Dilbone DA, Pereira PN, Duarte WR, Geraldeli S, Delgado AJ. Abfraction lesions: etiology, diagnosis, and treatment options. Clin Cosmet Investig Dent. 2016;8:79-87.

20. Amaral SM, Abad EC, Maia KD, Weyne S, Oliveira MPRPB, Tunãs ITC. Not carious lesions: the challenge of the multidisciplinary diagnosis. Int. Arch Otorhinolaryngol. 2012;16(1):96-102.

21. Ganss C, Klimek J, Giese K. Dental erosion in children and adolescents-a cross-sectional and longitudinal investigation using study models. Community Dentistry and Oral Epidemiology. 2001;29(4):264-71.

22. Dugmore CR, Rock WP. The progression of tooth erosion in a cohort of adolescents of mixed ethnicity.International Journal of Paediatric Dentistry. 2003;13(5):295-303.

23. El Aidi H, Bronkhorst EM, Huysmans MC, Truin GJ. Dynamics of tooth erosion in adolescents: a 3-year longitudinal study. Journal of Dentistry. 2010;38(2):131-7.

24. Dugmore CR, Rock WP. The progression of tooth erosion in a cohort of adolescents of mixed ethnicity. Int J Paediatr Dent. 2003;13(5):295-303.

25. Al-Dlaigan YH, Shaw L, Smith A. Dental erosion in a group of British 14-year old, schoolchildren. Part I: Prevalence and influence of differing socioeconomic backgrounds. BDJ. 2001;190(3):145-9.

26. Ren YF. Dental Erosion: Etiology, Diagnosis and Prevention. ADA CERP. 2011;76-84.

27. Daly B, Newton JT, Fares J, Chiu K, Ahmad N, Shirodaria S, et al. Dental tooth surface loss and quality of life in university students. Primary Dental Care. 2011;18(1):31-5.

28. Mathew T, Casamassimo PS, Hayes JR. Relationship between sports drinks and dental erosion in 304 university athletes in Columbus, Ohio, USA. Caries Res. 2002;36(4):281-7.

29. Hooper SM, Hughes JA, Newcombe RG, Addy M, West NX. A Methodology for Testing the Erosive Potential of Sports Driks. J Dent. 2005;33(4):343-8.

30. Ashley P, Di Iorio A, Cole E, Tanday A, Needleman I. Oral health of elite athletes and association with performance: a systematic review. Br J Sports Med. 2015;49(1):14-9.

31. Needleman I, Ashley P, Petrie A, Fortune F, Turner W, Jones J, et al. Oral health and impact on performance of athletes participating in the London 2012 Olympic Games: a cross-sectional study. Br J Sports Med. 2013;47(16):1054-8.

32. Lendenmann U, Grogan J, Oppenheim FG. Saliva and dental pellicle--a review. Adv Dent Res. 2000;14:22-8.
33. Holbrook WP, Furuholm J, Gudmundsson K, Theodórs A, Meurman JH. Gastric reflux is a Significant Causative Factor of Tooth Erosion. J Dent Res. 2009;88(5):422-6.

34. Hidalgo MG. Electromyography Evaluations of the masticator muscles during the maximum bite force. Rev Esp Cirug Oral y Maxilofac. 2008;30(6):428-30. 35. Rodrigues AMM, Berzin FS, Vania CV. Electromyographic analysis of the masseter and temporal muscles in posterior crossbite correction. Rev. Dent. Press Ortodon. Ortop. Facial. 2006;11(3):55-62.

36. Vougiouklakis G, Tzoutzas J, Farmakis ET, Farmakis EE, Antoniadou M, Mitsea A. Dental data of the Athens 2004 Olympic and Paralympic Games. Int J Sports Med. 2008;29(11):927-33.

37. Pack S, Kelly S, Arvinen-Barrow M. "I think I became a swimmer rather than just someone with a disability swimming up and down:" paralympic athletes perceptions of self and identity development. Disabil Rehabil. 2016:27:1-8.

38. Bartlett DW. The role of erosion in tooth wear: aetiology, prevention and management. Int. Dent. J. 2005;55(4):suppl1,277-84.

39. Pegoraro LF, Scolaro JM, Conti C, Telles D, Pegoraro TA. Noncarious cervical lesions inadults. J. Am. Dent. Assoc. 2005;136(12):1694-700.

40. Bishop NC, Blannin AK, Armstrong E, Rickman M, Gleeson M. Carbohydrate and fluid intake affect the saliva flow rate and IgA response to cycling. Med Sci Sports Exerc. 2000;32:2046-51.

41. Libicz S, Mercier B, Bigou N, Le Gallais D, Castex F. Salivary IgA response of triathletes participating in the French Iron Tour. Int J Sports Med. 2006;27(5):389-94.

42. Carpenter GH, Proctor GB, Anderson LC, Zhang XS, Garrett JR. Immunoglobulin A secretion into saliva during dual sympathetic and parasympathetic nerve stimulation of rat submandibular glands. Exp Physiol. 2000;85(3):281-6.

43. Cabrera AR, Kamashiro CR. Efecto Del pH Del água de piscina em esmalte de dientes decíduos humanos. Estúdio com microscopia eletrônica de barrido. Rev. Estomatol Herediana. 2004;14(1-2):59-62.

44. International Non-Toxic Water-Treatment Association (INWA). Swimming pool issues: version 1.0. 2005. Kuranda. Austrália.

45. Jensdottir T, Nauntofte B, Buchwald C, Bardow A. Effects of calcium on the erosive potential of acidic candies in saliva. Caries Res. 2007;41(1):68-73.

46. Amaechi BT, Higham SM. Dental erosion: possible approaches to prevention and control. J Dent. 2005;33(3):243-52.

47. Vieira A, Jager DH, Ruben JL, Huysmans MC. Inhibition of erosive wear by fluoride varnish. Caries Res. 2007;41(1):61-7.

\section{Mini Currículo e Contribuição dos Autores}

1. Bárbara Capitanio de Souza - cirurgiã-dentista e mestre.

Recebido em: 08/04/2017 / Aprovado em: 08/05/2017

Autora Correspondente

Bárbara Capitanio de Souza

E-mail: barbara.capitanio@gmail.com 